\title{
Highly Diastereoselective Approach toward ( \pm )-Tetraponerine T6 and Analogues via the Double Cycloisomerization-Reduction of Bis-alkynylpyrimidines
}

\author{
Joseph T. Kim, Jason Butt, and Vladimir Gevorgyan \\ Department of Chemistry, University of Illinois at Chicago, 875 West Taylor Street, Chicago, \\ Illinois 60607-7061 \\ Vladimir Gevorgyan: vlad@uic.edu
}

\begin{abstract}
A new, short, and efficient approach toward tricyclic alkaloids, involving the double cycloisomerization-reduction of bisalkynylpyrimidines $\mathbf{3 a}-\mathbf{m}$, has been developed. The requisite bis-alkynylpyrimidines 3a-m were readily prepared via regioselective sequential Sonogashira coupling reactions of dibromopyrimidines $\mathbf{1}$. Bis-alkynylpyrimidines $\mathbf{3} \mathbf{a}-\mathbf{m}$ were converted into the 5-6-5 tricyclic heteroaromatic cores $\mathbf{4 a}-\mathbf{m}$ via the $\mathrm{Cu}(\mathrm{I})$-assisted double cycloisomerization reaction. The reaction proceeded stepwise, which was confirmed by the isolation of the monopyrrolization intermediate $\mathbf{5}$. The structure of $\mathbf{5}$ was assigned by 2D NMR and by independent synthesis. Cycloisomerization of $\mathbf{5}$ under standard conditions afforded tricyclic $\mathbf{4 g}$ in $89 \%$ yield. The $\mathrm{PtO}_{2}$-catalyzed hydrogenation of bis-pyrrolopyrimidines $\mathbf{4 d}, \mathbf{4 g}$, and $\mathbf{4 i}$ in acidic media afforded stable amidinium derivatives, 11a, 11b, and 11c. Further reduction of the latter with $\mathrm{LiAlH}_{4}$ allowed for the highly diastereoselective total synthesis of ( \pm )-tetraponerine T6 and its analogues.
\end{abstract}

\section{Introduction}

The tetraponerines are a group of eight toxic alkaloids that are found in the venom of the New Guinean pseudomyrmecine ant Tetraonera $s p{ }^{1}$ and are composed of 1,3-diaza tricyclic systems (5-6-5 and 6-5-6), highly unusual cores for alkaloids isolated from animals.

Moreover, these alkaloids represent the major constituents of the contact poison, showing profound insecticidal activities ( $\mathrm{LD}_{50}$ of $5 \times 10^{-9} \mathrm{~mol} / \mathrm{ant} \mathrm{mg}$ ). The unprecedented tricyclic skeleton, along with the interesting insecticidal activities of the tetraponerines, has made them attractive targets for total synthesis. Tetraponerine T6 is made of a 5-6-5 skeleton and is one of the major venom alkaloids. Prior to our studies, four diastereo- and enantioselective syntheses of tetraponerine T6 had been reported. ${ }^{2}$ These syntheses, representing two synthetic approaches toward assembling a tricyclic core of tetraponerine T6, are summarized in Scheme 1. Blechert assembled the tricyclic 5-6-5 core via a cascade Pd-catalyzed cyclization. ${ }^{2 a}$ Another approach, utilized by Plehiers, ${ }^{2 b}$ Royer, ${ }^{2 c}$ and Devijver, ${ }^{2 \mathrm{~d}}$ employed different modes of double condensation of proline homologues with cyclic imines. We have recently communicated a short and highly diastereoselective synthesis of $( \pm)$-tetraponerine T6, employing a totally different approach ${ }^{3}$ involving exhaustive hydrogenation/reduction of the bispyrrolopyrimidine skeleton obtained via the

(C) 2004 American Chemical Society

Correspondence to: Vladimir Gevorgyan, vladeuic .edu.

Supporting Information Available: General information and copies of ${ }^{1} \mathrm{H}$ and ${ }^{13} \mathrm{C}$ NMR spectra for $\mathbf{4 f}-\mathbf{m}, \mathbf{5}, \mathbf{1 2 a}$, and 12c. Copies of NOESY spectra for 12a and 12c. This material is available free of charge via the Internet at http://pubs.acs.org. 
double cycloisomerization of bisalkynylpyrimidine (Scheme 1). This strategy represents a conceptually novel and highly expeditious route toward certain polycyclic alkaloid skeletons. Herein, we provide a full account of this work as well as additional mechanistic studies and syntheses of tetraponerine analogues.

\section{Results and Discussion}

Recently, we developed a novel, general, and efficient method for the construction of pyrrole rings and fused aromatic pyrroloheterocycles via the $\mathrm{Cu}$-assisted cycloisomerization of alkynyl imines. ${ }^{4}$ The synthetic usefulness of this novel methodology was further demonstrated by achieving the shortest synthesis of $( \pm)$-monomorine in three steps and $47 \%$ overall yield (Scheme 2). This successful result encouraged us to investigate the prospect of multiple pyrrolization protocols en route to tricyclic alkaloid structures. To test this idea, we investigated the possibility of constructing a 5-6-5 tricyclic heteroaromatic skeleton via the double $\mathrm{Cu}(\mathrm{I})$-assisted cycloisomerization of bis-alkynylpyrimidines.

To this end, we synthesized various bis-alkynylpyrimidine derivatives $\mathbf{3 a} \mp \mathbf{m}$ employing Sonogashira coupling reactions ${ }^{5}$ of 1,3-dibromopyrimidines 1 (Table 1). Double-fold Sonogashira coupling of dibromopyrimidines ${ }^{6}$ with propyne proceeded smoothly to give bispropynylpyrimide derivatives in quantitative yields (Table 1, entries 1-5). Analogously, employment of higher alkyne analogues, such as pentyne and hexyne, allowed for the efficient syntheses of symmetric bis-alkynylpyrimidines 3ffi (Table 1, entries 6-9). Next, we synthesized bisalkynylpyrimidines $\mathbf{3} \mathbf{j}-\mathbf{m}$, possessing different alkynyl substituents (Table 1, entries 10-13). Here, we took advantage of the known, different reactivity of bromides in dibromopyrimidines $\mathbf{1} .^{7}$ First, we accessed bisalkynylpyrimidines $\mathbf{3 j}-\mathbf{l}$ in stepwise fashion. Accordingly, employment of 1 equiv of alkyne allowed for selective coupling at the C-4 position to give the corresponding bromopyrimidine 2 in reasonable to good yields. Subsequent coupling of the $\mathrm{C}-2$ bromide with another alkyne provided the unsymmetrical bisalkynylpyrimidines in excellent overall yield (Table 1, entries 10-12). It was also found that the cross-coupling of $\mathbf{1}$ with two different alkynes can efficiently be performed under a one-pot procedure condition without isolation of monobromopyrimidine intermediate 2 (Table 1, entry 13).

Next, bis-alkynylpyrimidine 3d, a potential precursor of tetraponerine T6, was chosen for optimization of the double cycloisomerization process (Table 2). The attempts at double cycloisomerization of $\mathbf{3 d}$ in the presence of 2 equiv of $\mathrm{CuI}$ at various temperatures were unsuccessful; at higher temperatures (Table 2, entries 1,2), the substrate polymerized, whereas at lower temperatures (Table 2, entry 3), no reaction occurred. Reduction of the CuI amount had a somewhat positive effect on the reaction course (Table 2, entries 4-6). Further improvement was achieved by switching to $\mathrm{CuBr}$ (Table 2, entry 9). Dilution of the reaction mixture allowed for additional improvement of the reaction yields (Table 2, entries 10,11). Taking into account that the yield for each pyrrolization in the transformation of $\mathbf{3 d}$ to $\mathbf{4 d}$ is about $70 \%$ and that the cycloisomerization yields for propyne derivatives are normally 10 $20 \%$ lower than that of their higher homologues, ${ }^{8}$ we considered $50-52 \%$ yield for the double pyrrolization to be a rather satisfactory result.

The optimized conditions were applied to the double cycloisomerization of the differently substituted bisalkynylpyrimidines (Table 3). It was found that the double cycloisomerization of bisalkynylpyrimidines generally allowed for the assembly of tricyclic aromatic compounds in moderate to good yields. Double cycloisomerization of $\mathbf{3 a}$ provided low yield of nonsubstituted bis-pyrrolopyrimidine $\mathbf{4 a}$ (Table 3, entry 1). Notably, introduction of additional alkyl substituents into bis-alkynylpyrimidines had a positive effect on the yields of the resulting bis-pyrrolopyrimidines, ranging from moderate yields for mono-, di-, and 
trisubstituted heterocycles (Table 3, entries 2-8) to good yields for tetra-alkyl-substituted heterocycles (Table 3, entries 9, 12,13). For reasons which are not completely understood, the introduction of a methoxy substituent $\left(\mathrm{R}^{3}\right.$ or $\left.\mathrm{R}^{4}\right)$ led to substantial decrease in the reaction yields (Table 3 , entries 10,11 ).

Apparently, the double cycloisomerization of bis-alkynylpyrimidines proceeds via an alternative sequence of single cycloisomerization steps. As depicted in Scheme 3, the first pyrrolization of bis-alkynylpyrimidine $\mathbf{3 g}$ can proceed in three possible ways (Scheme 3, paths A-C). Among them, paths A and B, after the first cycloisomerization, will produce pyrrolopyrimidines $\mathbf{5}$ and $\mathbf{6}$, which, after the second pyrrolization, will give rise to the desired product $\mathbf{4 g}$. In contrast to these cases, path $\mathrm{C}$ leads to the dead-end intermediate $\mathbf{7 .}$ Under standard reaction conditions ( 1 equiv of $\mathrm{CuBr}$ in dilute $\mathrm{Et}_{3} \mathrm{~N}-\mathrm{DMA}$ at $150^{\circ} \mathrm{C}$ ), no other low-molecular-weight compounds, besides $\mathbf{4 g}$, were detected by GC-MS analyses of the crude reaction mixture. In contrast, when the reaction was performed at both reduced temperature $\left(130^{\circ} \mathrm{C}\right)$ and copper loading $(30 \mathrm{~mol} \%)$, GC-MS analysis revealed the presence of one isomeric compound along with starting material $\mathbf{3 g}$ and product $\mathbf{4 g}$. This intermediate had quite a different $R_{f}$ value from those of starting material $3 \mathbf{g}$ or product $\mathbf{4 g}$, and thus it was easily separated by column chromatography and its structure assigned as $\mathbf{5}$ on the basis of extensive 2D NMR studies, including COSY, heteronuclear multiplequantum coherence (HMQC), and heteronuclear multiple-bond correlation (HMBC). The structure of intermediate $\mathbf{5}$ was additionally proven by an independent synthesis, depicted in Scheme 4. Thus, known dichloropyrimidine 8 (Scheme 4) was first coupled with 1 equiv of hexyne under mild Sonogashira reaction conditions to give mono-alkynylated pyrimidine $\mathbf{9}$, which was cycloisomerized into mono-pyrrolopyrimidine 10. The subsequent hightemperature Sonogashira coupling of $\mathbf{1 0}$ with another molecule of hexyne produced $\mathbf{5}$, which was identical to the isolated intermediate in all respects. When $\mathbf{5}$ was subjected to the cycloisomerization conditions, it was smoothly converted into $\mathbf{4 g}$ in $89 \%$ yield (Scheme 4 ).

Next, we drew our attention to the exhaustive reduction of bispyrrolopyrimidines (Table 4). Direct, complete hydrogenation of heteroaromatic compound 4d to 12a proved not to be straightforward. It is well-known that catalytic hydrogenation of pyrimidine derivatives in acidic media is cis-diastereoselective and stops at the stage of formation of stable amidinium derivatives. ${ }^{9}$ Accordingly, catalytic hydrogenation of $\mathbf{4 d}$ over $\mathrm{PtO}_{2}$ under acidic conditions gave stable amidinium salt 11a as a single cis-isomer. ${ }^{10}$ The total synthesis of $( \pm)$ tetraponerine $\mathrm{T} 6$ was completed by a highly diastereo-selective reduction of crude 11a with $\mathrm{LiAlH}_{4}$ to give 12a (T6) as the sole stereoisomer in 64\% yield over two steps.

Exhaustive hydrogenation/reduction of trisubstituted bis-pyrrolopyrimidine $\mathbf{4 g}$ and the tetrasubstituted analogue $\mathbf{4 i}$ proceeded with high diastereoselectivity, producing all-cismultisubstituted tetraponerine analogues $\mathbf{1 2 b}-\mathbf{c}$ in 88:12 diastereomeric ratios with a minor unidentified diastereomer. Notably, tetraponerine 12a and its tetra-substituted analogue 12c are very stable compounds. In contrast, $\mathbf{1 2 b}$, possessing slightly longer side chains, appeared to be extremely unstable and was characterized as crude. Diastereomeric purity of each compound was determined by ${ }^{1} \mathrm{H}$ NMR and GC-MS analyses of the crude reaction mixtures.

The highly diastereoselective installation of the last stereogenic center at C-2 via the reduction of 11a, which we believe is both sterically and stereoelectronically controlled, deserves a special note. ${ }^{11}$ Our molecular mechanics force field (MMFF) calculations predicted that delivery of the hydride can be sterically controlled, favoring $\beta$-face attack at the most stable amidinium ion conformer with alkyl substituents occupying the equatorial position (11a-eq), as well as at another conformer 11a-ax (Figure 1). Stereoelectronic reasons can also account for the delivery of hydride to the $\mathrm{C}-2$ of the amidinium ion. The 
nucleophilic attack by the hydride proceeds from the $\beta$-face to give ( \pm )-T6 through the most favorable chairlike transition state $\mathbf{i}$, instead of an a-face delivery of a hydride through the disfavored boatlike transition state ii to form an epi-( \pm )-T6 (Figure 2). The relative configurations of $( \pm)$-tetraponerine T6 and its analogues were confirmed by NOESY and ${ }^{1} \mathrm{H}$ NOE experiments.

\section{Conclusions}

In summary, the $\mathrm{Cu}(\mathrm{I})$-assisted double pyrrolization of bis-alkynylpyrimidine to the 5-6-5 heteroaromatic core was demonstrated. A highly selective hydrogenation/reduction of the resulting bis-pyrrolopyrimidine allowed for the short, efficient, and highly diastereoselective total synthesis of $( \pm)$-tetraponerine T6 and its analogues. Considering that the assembly of a 5-6-5 tricyclic skeleton by this double pyrrolization-reduction functionalization protocol allows for the quick installation of up to 6 stereo centers in a highly diastereoselective manner, this method can serve as a new, short, and efficient approach toward selected polycylic alkaloid structures.

\section{Experimental Section}

All manipulations were conducted under argon atmosphere using a combination of glovebox and standard Schlenk techniques. Anhydrous $\mathrm{Et}_{3} \mathrm{~N}$ and DMA were purchased from Aldrich and stored over calcium hydride. Anhydrous THF and benzene were distilled over sodium/ benzophenone. 6-Pentyl-1 $H$-pyrimidine-2,4-dione was prepared according to the known procedures. ${ }^{12}$

\section{Representative Procedure for Syntheses of Dibromopyrimidines (1, $\mathbf{R}_{\mathbf{1}}=\mathrm{CH}_{\mathbf{3}}, \mathbf{R}_{\mathbf{2}}=\mathbf{C H}_{\mathbf{3}}$ )}

5,6-Dimethyl-2,4-(1H,3H)-pyrimidinedione (2.08 g, $14.84 \mathrm{mmol}$ ), potassium carbonate $(6.15 \mathrm{~g}, 44.50 \mathrm{mmol})$, and phosphorus oxybromide $(12.76 \mathrm{~g}, 44.50 \mathrm{mmol})$ were heated to reflux in dry acetonitrile $(100 \mathrm{~mL})$ for $72 \mathrm{~h}$. The mixture was cooled to room temperature, poured into ice, and neutralized (solid potassium carbonate). The aqueous phase was thoroughly extracted with $\mathrm{CH}_{2} \mathrm{Cl}_{2}$. The combined organic extracts were washed (brine), dried (anhydrous $\mathrm{Na}_{2} \mathrm{SO}_{4}$ ), and concentrated under reduced pressure. The residue was purified by silica gel chromatography with $10 \%$ EtOAc/hexanes to give 2,4-dibromo-5,6dimethyl-pyrimidine as a solid with $\mathrm{mp} 125^{\circ} \mathrm{C}(3.28 \mathrm{~g}, 83 \%)$.

\section{Representative Procedure for Mono-Sonogashira Reaction 2j}

The mixture of 2,4-dibromo-5,6-dimethylpyrimidine (580 mg, $2.18 \mathrm{mmol}$ ), CuI (17 mg, $0.09 \mathrm{mmol})$, and $\mathrm{Pd}\left(\mathrm{PPh}_{3}\right)_{2}-\mathrm{Cl}_{2}(31 \mathrm{mg}, 0.04 \mathrm{mmol})$ was stirred at room temperature, and then pent-4-ynyl-bezene $(280 \mu \mathrm{L}, 2.43 \mathrm{mmol})$ was added to the mixture and stirred for $12 \mathrm{~h}$. The mixture was quenched (aqueous $\mathrm{NH}_{4} \mathrm{Cl}$ ). The phases were separated, and the aqueous phase was thoroughly extracted with hexanes. The combined organic extracts were washed (brine), dried (anhydrous $\mathrm{Na}_{2}-\mathrm{SO}_{4}$ ), and concentrated under reduced pressure. The residue was purified by silica gel chromatography with $5-10 \%$ EtOAc/hexanes to give $\mathbf{2 j}$ as an oil (574 mg, 80\%).

2j. ${ }^{1} \mathrm{H}$ NMR (500 MHz, $\left.\mathrm{CDCl}_{3}, \delta\right): 7.30-7.18(5 \mathrm{H}, \mathrm{m}), 2.77(2 \mathrm{H}, \mathrm{t}, J=7.6 \mathrm{~Hz}), 2.50(2 \mathrm{H}, \mathrm{t}$, $J=7.1 \mathrm{~Hz}), 2.48(3 \mathrm{H}, \mathrm{s}), 2.32(3 \mathrm{H}, \mathrm{s}), 1.97(2 \mathrm{H}$, quint, $J=7.5 \mathrm{~Hz}) .{ }^{13} \mathrm{C} \mathrm{NMR}(125 \mathrm{MHz}$, $\left.\mathrm{CDCl}_{3}, \delta\right): 168.9,152.0,148.9,141.0,129.4,128.5(\times 4), 126.1,100.7,77.7,34.9,29.6$, 22.5, 19.0, 15.0. MS m/ $z$ (relative intensity): 329 ( $\left.\mathrm{M}^{+}, 14\right), 300$ (27), 224 (100), 91 (89).

2k. ${ }^{1} \mathrm{H}$ NMR (500 MHz, $\mathrm{CDCl}_{3}, \delta$ ): 4.32 (2H, s), 3.39 (3H, s), $2.44(3 \mathrm{H}, \mathrm{s}), 2.29$ (3H, s). ${ }^{13} \mathrm{C} \mathrm{NMR}\left(125 \mathrm{MHz}, \mathrm{CDCl}_{3}, \delta\right): 169.4,150.8,148.9,129.7,94.5,82.0,60.1,58.1,22.5$, 14.9. MS m/z (relative intensity): $255\left(\mathrm{M}^{+}, 1\right), 224$ (100), 143 (48), 77 (62). 


\section{Representative Procedure for Syntheses of Di-prop-1-ynyl-pyrimidine (3c)}

Using a high-pressure tube, propyne $(10 \mathrm{~mL})$ was condensed in a mixture of 2,4-dibromo-5methylpyrimidine ( $2.50 \mathrm{~g}, 9.92 \mathrm{mmol})$, $\mathrm{CuI}$ (76 mg, $0.4 \mathrm{mmol}), \mathrm{Pd}-\left(\mathrm{PPh}_{3}\right)_{2} \mathrm{Cl}_{2}(140 \mathrm{mg}, 0.2$ $\mathrm{mmol})$, and $\mathrm{Et}_{3} \mathrm{~N}(50 \mathrm{~mL})$ at $-78{ }^{\circ} \mathrm{C}$. The mixture was slowly warmed and stirred at $50{ }^{\circ} \mathrm{C}$ for $3 \mathrm{~h}$. Then, the mixture was cooled to room temperature and quenched (aqueous $\mathrm{NH}_{4} \mathrm{Cl}$ ). The phases were separated, and the aqueous phase was thoroughly extracted with hexanes. The combined organic extracts were washed (brine), dried (anhydrous $\mathrm{Na}_{2} \mathrm{SO}_{4}$ ), and concentrated under reduced pressure. The residue was purified by silica gel chromatography with $20 \%$ EtOAc/hexanes to give $\mathbf{3 c}$ as a solid $(1.69 \mathrm{~g},>99 \%)$.

\section{Representative Procedure for Sequential Sonogashira Reaction (3m)}

The mixture of 2,4-dibromo-5,6-dimethyl-pyrimidine ( $380 \mathrm{mg}, 1.43 \mathrm{mmol}$ ), CuI (11 mg, $0.06 \mathrm{mmol})$, and $\mathrm{Pd}\left(\mathrm{PPh}_{3}\right)_{2} \mathrm{Cl}_{2}(20 \mathrm{mg}, 0.03 \mathrm{mmol})$ was stirred at room temperature, and then $n$-hexyne $(173 \mu \mathrm{L}, 1.5 \mathrm{mmol})$ was added to the mixture and stirred for $12 \mathrm{~h}$. The reaction progress was monitored by TLC and GC-MS analyses. Pent-4-ynyl-benzene (430 $\mu \mathrm{L}, 2.83 \mathrm{mmol}$ ) was added to the mixture and stirred for $12 \mathrm{~h}$ at $45^{\circ} \mathrm{C}$. The mixture was cooled to room temperature and quenched (aqueous $\mathrm{NH}_{4} \mathrm{Cl}$ ). The phases were separated, and the aqueous phase was thoroughly extracted with hexanes. The combined organic extracts were washed (brine), dried (anhydrous $\mathrm{Na}_{2} \mathrm{SO}_{4}$ ), and concentrated under reduced pressure. The residue was purified by silica gel chromatography with 2-10\% EtOAc/ hexanes to give $\mathbf{3 m}$ as an oil (355 $\mathrm{mg}, 75 \%)$.

3a. $\mathrm{Mp} 58{ }^{\circ} \mathrm{C} .{ }^{1} \mathrm{H}$ NMR $\left(500 \mathrm{MHz}, \mathrm{CDCl}_{3}, \delta\right): 8.49(1 \mathrm{H}, \mathrm{d}, J=5.1 \mathrm{~Hz}), 7.08(1 \mathrm{H}, \mathrm{d}, J=5.1$ $\mathrm{Hz}), 2.00(3 \mathrm{H}, \mathrm{s}), 1.99(3 \mathrm{H}, \mathrm{s}) .{ }^{13} \mathrm{C} \mathrm{NMR}\left(125 \mathrm{MHz}, \mathrm{CDCl}_{3}, \delta\right): 157.5,153.5,151.8,121.6$, 93.2, 86.9, 79.4, 78.2, 4.8, 4.6. MS m/ $z$ (relative intensity): $156\left(\mathrm{M}^{+}, 100\right), 128$ (5), 91 (14), 64 (52).

3b. Mp $68{ }^{\circ} \mathrm{C} .{ }^{1} \mathrm{H}$ NMR $\left(500 \mathrm{MHz}, \mathrm{CDCl}_{3}, \delta\right): 6.88(1 \mathrm{H}, \mathrm{s}), 2.29(3 \mathrm{H}, \mathrm{s}), 1.90(3 \mathrm{H}, \mathrm{s}), 1.89$ $(3 \mathrm{H}, \mathrm{s}) .{ }^{13} \mathrm{C} \mathrm{NMR}\left(125 \mathrm{MHz}, \mathrm{CDCl}_{3}, \delta\right): 167.8,152.9,151.3,121.1,92.2,86.3,79.3,78.2$, 24.2, 4.7, 4.5. MS m/ $z$ (relative intensity): $170\left(\mathrm{M}^{+}, 100\right), 78$ (14), 64 (38).

3c. Mp $143{ }^{\circ} \mathrm{C} .{ }^{1} \mathrm{H}$ NMR $\left(500 \mathrm{MHz}, \mathrm{CDCl}_{3}, \delta\right): 8.44(1 \mathrm{H}, \mathrm{s}), 2.31(3 \mathrm{H}, \mathrm{s}), 2.12(3 \mathrm{H}, \mathrm{s}), 2.04$ $(3 \mathrm{H}, \mathrm{s}) .{ }^{13} \mathrm{C} \mathrm{NMR}\left(125 \mathrm{MHz}, \mathrm{CDCl}_{3}, \delta\right): 157.8,151.4,151.1,130.8,96.6,85.9,79.3,77.0$, 16.6, 5.0, 4.7. MS m/z (relative intensity): 170 ( $\left.\mathrm{M}^{+}, 100\right), 78$ (33), 66 (33).

3d. ${ }^{1} \mathrm{H}$ NMR (500 MHz, $\mathrm{CDCl}_{3}, \delta$ ): $6.99(1 \mathrm{H}, \mathrm{s}), 2.63(2 \mathrm{H}, \mathrm{t}, J=7.8 \mathrm{~Hz}), 2.03(3 \mathrm{H}, \mathrm{s}), 2.02$ $(3 \mathrm{H}, \mathrm{s}), 1.64(2 \mathrm{H}$, quint, $J=7.7 \mathrm{~Hz}), 1.28-1.25(4 \mathrm{H}, \mathrm{m}), 0.83(3 \mathrm{H}, \mathrm{t}, 3.2 \mathrm{~Hz}) .{ }^{13} \mathrm{C}$ NMR $(125$ $\mathrm{MHz}, \mathrm{CDCl}_{3}, \delta$ ): 171.8, 153.1, 151.4, 120.5, 92.0, 86.1, 79.5, 78.3, 38.0, 31.7, 28.9, 22.7, 14.2, 4.7, 4.6. MS m/z (relative intensity): $226\left(\mathrm{M}^{+}, 2\right), 197$ (8), 183 (17), 170 (100). Anal. Calcd for $\mathrm{C}_{15} \mathrm{H}_{18} \mathrm{~N}_{2}:$ C, 79.61; H, 8.02; N, 12.38. Found: C, 79.57; H, 7.98; N, 12.29.

3e. Mp $122{ }^{\circ} \mathrm{C} .{ }^{1} \mathrm{H}$ NMR $\left(500 \mathrm{MHz}, \mathrm{CDCl}_{3}, \delta\right): 2.36(3 \mathrm{H}, \mathrm{s}), 2.23(3 \mathrm{H}, \mathrm{s}), 2.02(3 \mathrm{H}, \mathrm{s}), 1.95$ $(3 \mathrm{H}, \mathrm{s}) .{ }^{13} \mathrm{C} \mathrm{NMR}\left(125 \mathrm{MHz}, \mathrm{CDCl}_{3}, \delta\right): 166.1,151.2,150.6,129.0,93.8,83.8,81.0,78.2$, 22.5, 15.1, 4.1, 3.8. MS m/z (relative intensity): $184\left(\mathrm{M}^{+}, 100\right), 142$ (14), 78 (52).

3f. ${ }^{1} \mathrm{H}$ NMR (500 MHz, $\left.\mathrm{CDCl}_{3}, \delta\right): 8.15(1 \mathrm{H}, \mathrm{d}, J=5.1 \mathrm{~Hz}), 7.11(1 \mathrm{H}, \mathrm{d}, J=5.1 \mathrm{~Hz}), 2.37-$ $2.33(4 \mathrm{H}, \mathrm{m}), 1.61-1.55(4 \mathrm{H}, \mathrm{m}), 0.99-0.94(6 \mathrm{H}, \mathrm{m}) .{ }^{13} \mathrm{C} \mathrm{NMR}\left(125 \mathrm{MHz}, \mathrm{CDCl}_{3}, \delta\right)$ : 157.0, 153.2, 151.5, 121.3, 97.0, 90.6, 79.9, 78.7, $21.4(\times 2), 21.3,21.2,13.6,13.5 . \mathrm{MS} \mathrm{m} / \mathrm{z}$ (relative intensity): $212\left(\mathrm{M}^{+}, 5\right), 184$ (100), 164 (11).

3g. ${ }^{1} \mathrm{H}$ NMR $\left(500 \mathrm{MHz}, \mathrm{CDCl}_{3}, \delta\right): 7.01(1 \mathrm{H}, \mathrm{s}), 2.65(2 \mathrm{H}, \mathrm{t}, J=7.8 \mathrm{~Hz}), 2.40(4 \mathrm{H}, \mathrm{t}, J=$ $7.3 \mathrm{~Hz}), 1.66-1.64(2 \mathrm{H}, \mathrm{m}), 1.60-1.55(4 \mathrm{H}, \mathrm{m}), 1.42(4 \mathrm{H}, \mathrm{sext}, J=7.5 \mathrm{~Hz}), 1.30-1.27(4 \mathrm{H}$, m), $0.90(3 \mathrm{H}, \mathrm{t}, J=7.4 \mathrm{~Hz}), 0.88(3 \mathrm{H}, \mathrm{t}, J=7.1 \mathrm{~Hz}), 0.85(3 \mathrm{H}, \mathrm{t}, J=7.1 \mathrm{~Hz}) \cdot{ }^{13} \mathrm{C}$ NMR $(125$ 
$\left.\mathrm{MHz}, \mathrm{CDCl}_{3}, \delta\right): 171.8,153.2,151.6,120.7,96.5,90.5,80.4,79.2,38.1,31.9,30.4(\times 2)$, 29.0, 22.8, 22.5, 22.4, 19.5, 19.4, 14.3, 14.0, 13.9. MS $\mathrm{m} / z$ (relative intensity): $310\left(\mathrm{M}^{+}, 2\right)$, 281 (10), 267 (16), 254 (100).

3h. ${ }^{1} \mathrm{H}$ NMR (500 MHz, $\left.\mathrm{CDCl}_{3}, \delta\right): 8.44(1 \mathrm{H}, \mathrm{s}), 2.45(2 \mathrm{H}, \mathrm{t}, J=7.0 \mathrm{~Hz}), 2.38(2 \mathrm{H}, \mathrm{t}, J=$ $7.0 \mathrm{~Hz}), 2.31(3 \mathrm{H}, \mathrm{s}), 1.66-1.61(4 \mathrm{H}, \mathrm{m}), 1.03(3 \mathrm{H}, \mathrm{t}, J=7.3 \mathrm{~Hz}), 1.01(3 \mathrm{H}, \mathrm{t}, J=7.3$ Hz). ${ }^{13} \mathrm{C} \mathrm{NMR}\left(125 \mathrm{MHz}, \mathrm{CDCl}_{3}, \delta\right): 157.7,151.6,151.2,130.8,101.1,90.0,80.2,77.9$, 44.3, 22.0, 21.9, 21.6, 16.7, 14.1, 14.0. MS $\mathrm{m} / z$ (relative intensity): $226\left(\mathrm{M}^{+}, 8\right), 198$ (100), $181(9), 169(9)$.

3i. ${ }^{1} \mathrm{H}$ NMR (500 MHz, $\left.\mathrm{CDCl}_{3}, \delta\right): 2.41(3 \mathrm{H}, \mathrm{s}), 2.40(2 \mathrm{H}, \mathrm{t}, J=7.1 \mathrm{~Hz}), 2.34(2 \mathrm{H}, \mathrm{t}, J=7.1$ $\mathrm{Hz}), 2.29(3 \mathrm{H}, \mathrm{s}), 1.62-1.57(4 \mathrm{H}, \mathrm{m}), 0.99(3 \mathrm{H}, \mathrm{t}, J=7.4 \mathrm{~Hz}), 0.97(3 \mathrm{H}, \mathrm{t}, J=7.4 \mathrm{~Hz}) .{ }^{13} \mathrm{C}$ NMR (125 MHz, $\left.\mathrm{CDCl}_{3}, \delta\right): 166.4,150.7,150.4,129.1,99.6,89.3,80.3,78.3,22.9,22.0$, $21.9(\times 2), 21.6,15.6,14.1,14.0$. MS $\mathrm{m} / z$ (relative intensity): $240\left(\mathrm{M}^{+}, 8\right), 225$ (9), 212 (100), 195 (8).

3j. ${ }^{1} \mathrm{H}$ NMR (500 MHz, $\left.\mathrm{CDCl}_{3}, \delta\right): 7.29-7.17(5 \mathrm{H}, \mathrm{m}), 4.31(2 \mathrm{H}, \mathrm{s}), 3.43(3 \mathrm{H}, \mathrm{s}), 2.77(2 \mathrm{H}$, $\mathrm{t}, J=7.5 \mathrm{~Hz}), 2.48(2 \mathrm{H}, \mathrm{t}, J=7.1 \mathrm{~Hz}), 2.47(3 \mathrm{H}, \mathrm{s}), 2.37(3 \mathrm{H}, \mathrm{s}), 1.95(2 \mathrm{H}$, quint, $J=7.5$ Hz). ${ }^{13} \mathrm{C}$ NMR (125 MHz, $\left.\mathrm{CDCl}_{3}, \delta\right): 166.3,150.3,149.2,141.1,129.6,128.5(\times 4), 126.1$, 99.2, 84.9, 82.9, 78.1, 60.0, 57.9, 34.9, 29.7, 22.6, 19.0, 15.4. MS $\mathrm{m} / z$ (relative intensity): $318\left(\mathrm{M}^{+}, 23\right), 290$ (14), 214 (100), 91 (93).

3k. ${ }^{1} \mathrm{H}$ NMR (500 MHz, $\mathrm{CDCl}_{3}$, s): 7.21-7.08 (5H, m), $4.30(2 \mathrm{H}, \mathrm{s}), 3.38(3 \mathrm{H}, \mathrm{s}), 2.70(2 \mathrm{H}$, $\mathrm{t}, J=7.5 \mathrm{~Hz}), 2.41(3 \mathrm{H}, \mathrm{s}), 2.38(2 \mathrm{H}, \mathrm{t}, J=7.1 \mathrm{~Hz}), 2.30(3 \mathrm{H}, \mathrm{s}), 1.89(2 \mathrm{H}$, quint, $J=7.6$ $\mathrm{Hz}) .{ }^{13} \mathrm{C} \mathrm{NMR}\left(125 \mathrm{MHz}, \mathrm{CDCl}_{3}, \delta\right): 166.6,150.0,149.1,141.2,129.2,128.4(\times 2), 128.3$ $(\times 2), 125.9,92.7,88.9,82.7,80.0,60.1,58.0,34.8,29.5,22.6,18.7,15.3 . \mathrm{MS} \mathrm{m} / \mathrm{z}$ (relative intensity): $318\left(\mathrm{M}^{+}-1,19\right), 214$ (100), 91 (73).

31. ${ }^{1} \mathrm{H}$ NMR (500 MHz, $\left.\mathrm{CDCl}_{3}, \delta\right): 7.24-7.12(5 \mathrm{H}, \mathrm{m}), 2.72(2 \mathrm{H}, \mathrm{t}, J=7.5 \mathrm{~Hz}), 2.44(2 \mathrm{H}, \mathrm{t}$, $J=7.1 \mathrm{~Hz}), 2.42(3 \mathrm{H}, \mathrm{s}), 2.37(2 \mathrm{H}, \mathrm{t}, J=7.2 \mathrm{~Hz}), 2.30(3 \mathrm{H}, \mathrm{s}), 1.90(2 \mathrm{H}$, quint, $J=7.6 \mathrm{~Hz})$, $1.55(2 \mathrm{H}$, quint, $J=7.3 \mathrm{~Hz}), 1.40(2 \mathrm{H}$, sext, $J=7.3 \mathrm{~Hz}), 0.86(3 \mathrm{H}, \mathrm{t}, J=7.3 \mathrm{~Hz}) .{ }^{13} \mathrm{C}$ NMR $\left(125 \mathrm{MHz}, \mathrm{CDCl}_{3}, \delta\right): 166.1,150.1,149.9,141.1,128.8,128.4$ (×4), 126.0, 98.8, 89.4, 79.7, 78.2, 34.8, 30.0, 29.7, 22.5, 22.1, $18.9(\times 2), 15.3,13.6 . \mathrm{MS} \mathrm{m} / z$ (relative intensity): 330 $\left(\mathrm{M}^{+}, 35\right), 301(29), 226$ (90), 91 (100).

3m. ${ }^{1} \mathrm{H}$ NMR (500 MHz, $\left.\mathrm{CDCl}_{3}, \delta\right): 7.26-7.11(5 \mathrm{H}, \mathrm{m}), 2.72(2 \mathrm{H}, \mathrm{t}, J=7.6 \mathrm{~Hz}), 2.44(2 \mathrm{H}$, $\mathrm{t}, J=7.1 \mathrm{~Hz}), 2.42(3 \mathrm{H}, \mathrm{s}), 2.40(2 \mathrm{H}, \mathrm{t}, J=7.1 \mathrm{~Hz}), 2.29(3 \mathrm{H}, \mathrm{s}), 1.91(2 \mathrm{H}$, quint, $J=7.5$ $\mathrm{Hz}), 1.57(2 \mathrm{H}$, quint, $J=7.5 \mathrm{~Hz}), 1.44(2 \mathrm{H}$, sext, $J=7.6 \mathrm{~Hz}), 0.88(3 \mathrm{H}, \mathrm{t}, J=7.3 \mathrm{~Hz}) .{ }^{13} \mathrm{C}$ NMR (125 MHz, $\left.\mathrm{CDCl}_{3}, \delta\right): 166.0,150.3,149.9,141.3,128.6,128.4(\times 4), 125.9,99.4$, $88.5,80.2,77.7,34.9,30.1,29.7,29.5,22.6,19.2,18.7,15.2,13.5$. MS $\mathrm{m} / z$ (relative intensity): 229 ( $\left.\mathrm{M}^{+}-1,19\right), 239$ (11), 226 (100), 91 (49).

\section{Representative Procedure for Double Cycloisomerization Reaction (4I)}

Using a high-pressure tube, the mixture of compound 31 ( $370 \mathrm{mg}, 1.12 \mathrm{mmol})$ and $\mathrm{CuBr}$ $(161 \mathrm{mg}, 1.12 \mathrm{mmol})$ in $\mathrm{Et}_{3} \mathrm{~N}(6 \mathrm{~mL})$ and DMA $(40 \mathrm{~mL})$ was stirred at $150^{\circ} \mathrm{C}$ for $18 \mathrm{~h}$. The reaction was protected from the light by covering the flask with aluminum foil. Then, the mixture was cooled to room temperature and quenched (aqueous $\mathrm{NH}_{4}-\mathrm{Cl}$ ). The phases were separated, and the aqueous phase was thoroughly extracted with hexanes. The combined organic extracts were washed (brine), dried (anhydrous $\mathrm{Na}_{2} \mathrm{SO}_{4}$ ), and concentrated under reduced pressure. The residue was purified by silica gel chromatography with $5 \% \mathrm{EtOAc/}$ hexanes ( $1 \% \mathrm{Et}_{3} \mathrm{~N}$ was used for deactivation of the silica gel) to give $\mathbf{4 l}$ as a solid (278 $\mathrm{mg}$, $75 \%)$. 
4a. Mp $145^{\circ} \mathrm{C} .{ }^{1} \mathrm{H}$ NMR $\left(500 \mathrm{MHz}, \mathrm{CDCl}_{3}, \delta\right): 7.34-7.33(1 \mathrm{H}, \mathrm{m}), 7.23(1 \mathrm{H}, \mathrm{d}, J=7.6$ $\mathrm{Hz}), 6.86(1 \mathrm{H}, \mathrm{dd}, J=3.1,1.7 \mathrm{~Hz}), 2.42(3 \mathrm{H}, \mathrm{s}), 6.64(1 \mathrm{H}, \mathrm{dd}, J=3.7,2.8 \mathrm{~Hz}), 6.58(1 \mathrm{H}, \mathrm{d}$, $J=7.6 \mathrm{~Hz}), 6.48(1 \mathrm{H}, \mathrm{t}, J=3.3 \mathrm{~Hz}), 6.40(1 \mathrm{H}, \mathrm{dd}, J=3.7,1.3 \mathrm{~Hz}), 6.17-6.16(1 \mathrm{H}, \mathrm{m}) .{ }^{13} \mathrm{C}$ NMR (125 MHz, $\left.\mathrm{CDCl}_{3}, \delta\right): 129.0,126.1,118.9,113.2,112.4,112.1,110.1,103.6,103.3$, 88.1. MS m/ $z$ (relative intensity): $156\left(\mathrm{M}^{+}, 100\right), 129(14), 102(10)$. Anal. Calcd for $\mathrm{C}_{10} \mathrm{H}_{8} \mathrm{~N}_{2}$ : C, 76.90; H, 5.16; N, 17.94. Found: C, 76.94; H, 5.21; N, 17.91.

4b. Mp $45{ }^{\circ} \mathrm{C} .{ }^{1} \mathrm{H}$ NMR $\left(500 \mathrm{MHz}, \mathrm{CDCl}_{3}, \delta\right): 7.34(1 \mathrm{H}, \mathrm{d}, J=1.0 \mathrm{~Hz}), 6.91(1 \mathrm{H}, \mathrm{dd}, J=$ $3.1,1.6 \mathrm{~Hz}), 6.65(1 \mathrm{H}, \mathrm{dd}, J=3.5,2.8 \mathrm{~Hz}), 6.55(1 \mathrm{H}, \mathrm{t}, J=3.4 \mathrm{~Hz}), 6.43(1 \mathrm{H}, \mathrm{s}), 6.32(1 \mathrm{H}$, dd, $J=3.6,1.1 \mathrm{~Hz}), 6.25(1 \mathrm{H}, \mathrm{dd}, J=3.8,1.6 \mathrm{~Hz}), 2.47(3 \mathrm{H}, \mathrm{s}) .{ }^{13} \mathrm{C} \mathrm{NMR}(125 \mathrm{MHz}$, $\left.\mathrm{CDCl}_{3}, \delta\right): 129.4,126.9,126.8,112.3,112.2,110.0,109.4,101.9,101.5,88.5,18.3 . \mathrm{MS} m / z$ (relative intensity): $170\left(\mathrm{M}^{+}, 100\right), 155$ (24), 142 (10), 115 (7), 85 (10). Anal. Calcd for $\mathrm{C}_{11} \mathrm{H}_{10} \mathrm{~N}_{2}$ : C, 77.62; H, 5.92; N, 16.46. Found: C, 77.69; H, 5.95; N, 16.45.

4c. Mp $68{ }^{\circ} \mathrm{C} .{ }^{1} \mathrm{H}$ NMR $\left(500 \mathrm{MHz}, \mathrm{CDCl}_{3}, \delta\right): 7.37(1 \mathrm{H}, \mathrm{dd}, J=2.7,1.4 \mathrm{~Hz}), 7.07(1 \mathrm{H}, \mathrm{d}, J$ $=1.2 \mathrm{~Hz}), 6.83(1 \mathrm{H}, \mathrm{d}, J=1.4 \mathrm{~Hz}), 6.67(1 \mathrm{H}, \mathrm{dd}, J=3.7,2.8 \mathrm{~Hz}), 6.47(1 \mathrm{H}, \mathrm{t}, J=3.1 \mathrm{~Hz})$, $6.41(1 \mathrm{H}, \mathrm{dd}, J=3.7,1.4 \mathrm{~Hz}), 6.16(1 \mathrm{H}, \mathrm{d}, J=3.6 \mathrm{~Hz}), 2.28(3 \mathrm{H} . \mathrm{s}) .{ }^{13} \mathrm{C} \mathrm{NMR}(125 \mathrm{MHz}$, $\left.\mathrm{CDCl}_{3}, \delta\right): 128.5,128.4,116.6,113.5,112.2,111.9,111.5,109.6,102.2,87.5,15.4 . \mathrm{MS} \mathrm{m} / z$ (relative intensity): $170\left(\mathrm{M}^{+}, 100\right), 155$ (43), 142 (14), 115 (10), 85 (11). Anal. Calcd for $\mathrm{C}_{11} \mathrm{H}_{10} \mathrm{~N}_{2}$ : C, 77.62; H, 5.92; N, 16.46. Found: C, 77.38; H, 5.92; N, 16.46.

4d. Mp $53{ }^{\circ} \mathrm{C} .{ }^{1} \mathrm{H}$ NMR $\left(500 \mathrm{MHz}, \mathrm{CDCl}_{3}, \delta\right): 7.29(1 \mathrm{H}, \mathrm{dd}, J=2.4,1.2 \mathrm{~Hz}), 6.93(1 \mathrm{H}$, dd, $J=3.1,1.6 \mathrm{~Hz}), 6.59(1 \mathrm{H}, \mathrm{dd}, J=3.6,2.9 \mathrm{~Hz}), 6.50(1 \mathrm{H}, \mathrm{t}, J=3.5 \mathrm{~Hz}), 6.41(1 \mathrm{H}, \mathrm{s}), 6.28$ $(1 \mathrm{H}, \mathrm{dd}, J=3.6,1.3 \mathrm{~Hz}), 6.19(1 \mathrm{H}, \mathrm{dd}, J=3.8,1.6 \mathrm{~Hz}), 2.75(2 \mathrm{H}, \mathrm{t}, J=7.6 \mathrm{~Hz}), 1.77(2 \mathrm{H}$, quint, $J=7.6 \mathrm{~Hz}), 1.47-1.38(4 \mathrm{H}, \mathrm{m}), 0.94(3 \mathrm{H}, \mathrm{t}, J=7.1 \mathrm{~Hz}) .{ }^{13} \mathrm{C} \mathrm{NMR}\left(125 \mathrm{MHz}, \mathrm{CDCl}_{3}\right.$, o): 131.0, 129.4, 126.8, 112.2 (×2), 109.9, 109.3, 101.6, 100.7, 88.2, 32.0, 31.6, 26.9, 22.9, 14.4. MS m/ $z$ (relative intensity): $226\left(\mathrm{M}^{+}, 50\right), 197$ (7), 183 (14), 170 (100). Anal. Calcd for $\mathrm{C}_{15} \mathrm{H}_{18} \mathrm{~N}_{2}$ : C, 79.61; H, 8.02; N, 12.38. Found: C, 79.67; H, 8.03; N, 12.40 .

4e. Mp $116^{\circ} \mathrm{C} .{ }^{1} \mathrm{H}$ NMR $\left(500 \mathrm{MHz}, \mathrm{CDCl}_{3}, \delta\right): 7.34(1 \mathrm{H}, \mathrm{dd}, J=2.7,1.4 \mathrm{~Hz}), 6.90(1 \mathrm{H}, \mathrm{dd}$, $J=2.8,1.5 \mathrm{~Hz}), 6.64(1 \mathrm{H}, \mathrm{dd}, J=3.6,3.0 \mathrm{~Hz}), 6.50(1 \mathrm{H}, \mathrm{t}, J=3.4 \mathrm{~Hz}), 6.33(1 \mathrm{H}, \mathrm{dd}, J=$ 3.7, $1.3 \mathrm{~Hz}), 6.20(1 \mathrm{H}, \mathrm{dd}, J=3.7,1.2 \mathrm{~Hz}), 2.44(3 \mathrm{H}, \mathrm{s}), 2.30(3 \mathrm{H}, \mathrm{s}) .{ }^{13} \mathrm{C}$ NMR $(125 \mathrm{MHz}$, $\left.\mathrm{CDCl}_{3}, \delta\right): 129.0,128.9,123.0,112.6,112.0,109.4,109.3,107.9,100.9,87.8,14.1,13.6$. MS m/ $z$ (relative intensity): $184\left(\mathrm{M}^{+}, 100\right), 142$ (14), 115 (7), 104 (10), 78 (52). Anal. Calcd for $\mathrm{C}_{12} \mathrm{H}_{12} \mathrm{~N}_{2}: \mathrm{C}, 78.23 ; \mathrm{H}, 6.57 ; \mathrm{N}, 15.21$. Found: C, 78.41; H, 6.57; N, 15.20.

4f. ${ }^{1} \mathrm{H}$ NMR $\left(500 \mathrm{MHz}, \mathrm{C}_{6} \mathrm{D}_{6}, \delta\right): 6.58(1 \mathrm{H}, \mathrm{d}, J=7.6 \mathrm{~Hz}), 6.46(1 \mathrm{H}, \mathrm{d}, J=3.7 \mathrm{~Hz}), 6.44$ $(1 \mathrm{H}, \mathrm{d}, J=3.7 \mathrm{~Hz}), 6.27(1 \mathrm{H}, \mathrm{d}, J=5.0 \mathrm{~Hz}), 6.26(1 \mathrm{H}, \mathrm{s}), 6.23(1 \mathrm{H}, \mathrm{d}, J=3.7 \mathrm{~Hz}), 3.00(2 \mathrm{H}$, q, $J=7.4 \mathrm{~Hz}), 2.37(2 \mathrm{H}, \mathrm{q}, J=7.4 \mathrm{~Hz}), 1.29(3 \mathrm{H}, \mathrm{t}, J=7.4 \mathrm{~Hz}), 1.18(3 \mathrm{H}, \mathrm{t}, J=7.4 \mathrm{~Hz}) .{ }^{13} \mathrm{C}$ $\operatorname{NMR}\left(125 \mathrm{MHz}, \mathrm{C}_{6} \mathrm{D}_{6}, \delta\right): 131.2,128.5,125.9,124.6,114.6,109.1,105.8,102.9,102.5$, 89.7, 22.0, 19.2, 13.3, 12.5. MS m/z (relative intensity): 212 ( $\left.\mathrm{M}^{+}, 43\right), 197$ (100), 181 (16), 91 (21), 78 (52). HRMS (EI) calcd for $\mathrm{C}_{14} \mathrm{H}_{16} \mathrm{~N}_{2}\left(\mathrm{M}^{+}\right)$212.1314, found 212.1314.

4g. Mp $60{ }^{\circ} \mathrm{C} .{ }^{1} \mathrm{H}$ NMR $\left(500 \mathrm{MHz}, \mathrm{CDCl}_{3}, \delta\right): 6.28(1 \mathrm{H}, \mathrm{d}, J=3.9 \mathrm{~Hz}), 6.25(1 \mathrm{H}, \mathrm{s}), 6.24$ $(1 \mathrm{H}, \mathrm{d}, J=3.7 \mathrm{~Hz}), 6.21(1 \mathrm{H}, \mathrm{d}, J=3.9 \mathrm{~Hz}), 6.12(1 \mathrm{H}, \mathrm{d}, J=3.7 \mathrm{~Hz}), 3.30(2 \mathrm{H}, \mathrm{t}, J=7.5$ $\mathrm{Hz}), 2.90(2 \mathrm{H}, \mathrm{t}, J=7.4 \mathrm{~Hz}), 2.87(2 \mathrm{H}, \mathrm{t}, J=7.7 \mathrm{~Hz}), 1.80(2 \mathrm{H}, \mathrm{sext}, J=7.5 \mathrm{~Hz}), 1.72-1.63$ $(4 \mathrm{H}, \mathrm{m}), 1.44-1.35(4 \mathrm{H}, \mathrm{m}), 1.08(3 \mathrm{H}, \mathrm{t}, J=7.3 \mathrm{~Hz}), 1.03(3 \mathrm{H}, \mathrm{t}, J=7.3 \mathrm{~Hz}), 0.93(3 \mathrm{H}, \mathrm{t}, J=$ 7.1 Hz). ${ }^{13} \mathrm{C} \mathrm{NMR}\left(125 \mathrm{MHz}, \mathrm{CDCl}_{3}, \delta\right): 131.5,130.3,128.8,126.1,125.6,109.7,109.1$, $102.7,99.6,89.4,32.8,32.0,31.3,30.9,28.8,23.5,22.5,22.2,14.0(\times 2)$. MS $\mathrm{m} / z$ (relative intensity): $310\left(\mathrm{M}^{+}, 33\right), 281$ (100), 209 (26), 195 (23). HRMS (EI) calcd for $\mathrm{C}_{21} \mathrm{H}_{30} \mathrm{~N}_{2}$ $\left(\mathrm{M}^{+}\right) 310.2409$, found 310.2398 .

4h. Mp $55^{\circ} \mathrm{C} .{ }^{1} \mathrm{H}$ NMR $\left(500 \mathrm{MHz}, \mathrm{CDCl}_{3}, \delta\right): 6.95(1 \mathrm{H}, \mathrm{s}), 6.35(1 \mathrm{H}, \mathrm{d}, J=3.6 \mathrm{~Hz}), 6.32$ $(1 \mathrm{H}, \mathrm{d}, J=3.6 \mathrm{~Hz}), 6.25(1 \mathrm{H}, \mathrm{d}, J=2.3 \mathrm{~Hz}), 6.18(1 \mathrm{H}, \mathrm{s}), 3.19(2 \mathrm{H}, \mathrm{q}, J=3.4 \mathrm{~Hz}), 2.77(2 \mathrm{H}$, 
q, $J=7.4 \mathrm{~Hz}), 2.27(3 \mathrm{H}, \mathrm{s}), 1.44(3 \mathrm{H}, \mathrm{t}, J=7.4 \mathrm{~Hz}), 1.38(3 \mathrm{H}, \mathrm{t}, J=7.4 \mathrm{~Hz}) .{ }^{13} \mathrm{C}$ NMR $(125$ $\mathrm{MHz}, \mathrm{CDCl}_{3}, \delta$ ): $131.5,127.8,127.7,124.2,112.4,111.1,108.1,104.7,100.2,86.3,21.7$, 19.1, 15.1, 13.1, 12.3. MS m/ $z$ (relative intensity): $226\left(\mathrm{M}^{+}, 45\right), 211$ (100), 195 (19), 98 (17). HRMS (EI) calcd for $\mathrm{C}_{15} \mathrm{H}_{18} \mathrm{~N}_{2}\left(\mathrm{M}^{+}\right) 226.1470$, found 226.1484 .

4i. Mp $123{ }^{\circ} \mathrm{C} .{ }^{1} \mathrm{H}$ NMR $\left(500 \mathrm{MHz}, \mathrm{CDCl}_{3}, \delta\right): 6.31(1 \mathrm{H}, \mathrm{m}), 6.24(1 \mathrm{H}, \mathrm{s}), 6.20(1 \mathrm{H}, \mathrm{d}, J=$ $3.5 \mathrm{~Hz}), 3.16-3.10(4 \mathrm{H}, \mathrm{m}), 2.61(3 \mathrm{H}, \mathrm{s}), 1.44(3 \mathrm{H}, \mathrm{t}, J) 7.3 \mathrm{~Hz}), 1.36(3 \mathrm{H}, \mathrm{t}, J) 7.3 \mathrm{~Hz}) .{ }^{13} \mathrm{C}$ NMR $\left(125 \mathrm{MHz}, \mathrm{CDCl}_{3}, \delta\right): 130.9,129.8,128.3,127.3,123.7,108.4,108.9,107.7,99.2$, 88.7, 23.8, 22.1, 16.0, 14.2, 13.6, 13.1. MS m/ $z$ (relative intensity): $240\left(\mathrm{M}^{+}, 54\right), 225$ (100), 210 (21), 105 (24). HRMS (EI) calcd for $\mathrm{C}_{16} \mathrm{H}_{20} \mathrm{~N}_{2}\left(\mathrm{M}^{+}\right)$240.1627, found 240.1614.

4j. ${ }^{1} \mathrm{H}$ NMR (500 MHz, $\left.\left.\mathrm{CDCl}_{3}, \delta\right): 7.38-7.26(5 \mathrm{H}, \mathrm{m}), 6.28(1 \mathrm{H}, \mathrm{d}, J) 3.3 \mathrm{~Hz}\right), 6.16(1 \mathrm{H}, \mathrm{d}$, J) $3.6 \mathrm{~Hz}), 6.10(1 \mathrm{H}, \mathrm{s}), 5.56(1 \mathrm{H}, \mathrm{s}), 3.86(3 \mathrm{H}, \mathrm{s}), 3.37(2 \mathrm{H}, \mathrm{t}, J) 7.9 \mathrm{~Hz}), 3.11(2 \mathrm{H}, \mathrm{t}, J) 7.9$ $\mathrm{Hz}), 2.60(3 \mathrm{H}, \mathrm{s}), 2.19(3 \mathrm{H}, \mathrm{s}) .{ }^{13} \mathrm{C}$ NMR $\left(125 \mathrm{MHz}, \mathrm{CDCl}_{3}, \delta\right): 142.7,141.6,128.5,128.4$ (×4), 126.0, 123.1, 122.7, 109.0, 107.5, 99.5, 85.6, 85.1, 58.6, 35.1, 30.6, 14.8, 13.1. MS m/ $z$ (relative intensity): $318\left(\mathrm{M}^{+}, 26\right), 227$ (100), 212 (74), 183 (64), 91 (40). HRMS (EI) calcd for $\mathrm{C}_{21} \mathrm{H}_{22} \mathrm{~N}_{2} \mathrm{O}\left(\mathrm{M}^{+}\right)$318.1732, found 318.1738.

4k. Mp $122{ }^{\circ} \mathrm{C} .{ }^{1} \mathrm{H}$ NMR $\left(500 \mathrm{MHz}, \mathrm{CDCl}_{3}\right.$, o): 7.36-7.25 (5H, m), $6.48(1 \mathrm{H}, \mathrm{s}), 6.24(1 \mathrm{H}$, s), $6.06(1 \mathrm{H}, \mathrm{d}, J) 2.4 \mathrm{~Hz}), 5.73(1 \mathrm{H}, \mathrm{s}), 3.98(3 \mathrm{H}, \mathrm{s}), 3.35(2 \mathrm{H}, \mathrm{t}, J=8.1 \mathrm{~Hz}), 3.03(2 \mathrm{H}, \mathrm{t}, J=$ $8.1 \mathrm{~Hz}), 2.59(3 \mathrm{H}, \mathrm{s}), 2.17(3 \mathrm{H}, \mathrm{s}) .{ }^{13} \mathrm{C} \mathrm{NMR}\left(125 \mathrm{MHz}, \mathrm{CDCl}_{3}, \delta\right): 144.2,141.6,128.3$ (×4), 127.4, 126.0, 125.0, 122.7, 120.7, 108.6, 108.2, 97.0, 89.2, 87.4, 58.4, 36.5, 32.6, 16.0, 13.1. MS m/ $z$ (relative intensity): $318\left(\mathrm{M}^{+}, 23\right), 227$ (90), 212 (100), 183 (96), 91 (40). HRMS (EI) calcd for $\mathrm{C}_{21} \mathrm{H}_{22} \mathrm{~N}_{2} \mathrm{O}\left(\mathrm{M}^{+}\right) 318.1732$, found 318.1729.

4l. Mp $79{ }^{\circ} \mathrm{C} .{ }^{1} \mathrm{H}$ NMR (500 MHz, $\left.\mathrm{CDCl}_{3}, \delta\right): 7.46-7.34(5 \mathrm{H}, \mathrm{m}), 6.42(1 \mathrm{H}, \mathrm{d}, J=3.0 \mathrm{~Hz})$, $6.41(1 \mathrm{H}, \mathrm{s}), 6.31(1 \mathrm{H}, \mathrm{s}), 6.29(1 \mathrm{H}, \mathrm{d}, J=3.5 \mathrm{~Hz}), 3.50(2 \mathrm{H}, \mathrm{d}, J=7.9 \mathrm{~Hz}), 3.21(2 \mathrm{H}, \mathrm{d}, J=$ $7.9 \mathrm{~Hz}), 3.10(2 \mathrm{H}, \mathrm{d}, J=7.7 \mathrm{~Hz}), 2.67(3 \mathrm{H}, \mathrm{s}), 2.30(3 \mathrm{H}, \mathrm{s}), 1.82(2 \mathrm{H}, \mathrm{sext}, J=7.6 \mathrm{~Hz}), 1.14$ $(3 \mathrm{H}, \mathrm{t}, J=7.3 \mathrm{~Hz}) .{ }^{13} \mathrm{C}$ NMR $\left(125 \mathrm{MHz}, \mathrm{CDCl}_{3}, \delta\right): 141.5(\times 2), 129.5,128.4(\times 4), 125.9$, 125.7, 123.7 (×2), 109.5, 108.8, 108.4, 99.3, 88.6, 35.1, 32.7, 30.7, 23.4, 15.8, 14.0, 13.6. MS m/ $z$ (relative intensity): 330 ( $\mathrm{M}^{+}, 30$ ), 239 (100), 209 (19), 91 (9). HRMS (EI) calcd for $\mathrm{C}_{23} \mathrm{H}_{26} \mathrm{~N}_{2}\left(\mathrm{M}^{+}\right)$330.2096, found 330.2092.

4m. Mp $76{ }^{\circ} \mathrm{C} .{ }^{1} \mathrm{H}$ NMR $\left(500 \mathrm{MHz}, \mathrm{CDCl}_{3}, \delta\right): 7.41-7.30(5 \mathrm{H}, \mathrm{m}), 6.37(1 \mathrm{H}, \mathrm{d}, J=3.5 \mathrm{~Hz})$, $6.33(2 \mathrm{H}, \mathrm{s}), 6.25(1 \mathrm{H}, \mathrm{d}, J=3.4 \mathrm{~Hz}), 3.41(2 \mathrm{H}, \mathrm{d}, J=7.8 \mathrm{~Hz}), 3.12-3.06(4 \mathrm{H}, \mathrm{m}), 2.65(3 \mathrm{H}$, s), $2.26(3 \mathrm{H}, \mathrm{s}), 1.89(2 \mathrm{H}$, sext, $J=7.5 \mathrm{~Hz}), 1.17(3 \mathrm{H}, \mathrm{t}, J=7.3 \mathrm{~Hz}) .{ }^{13} \mathrm{C}$ NMR $(125 \mathrm{MHz}$, $\left.\mathrm{CDCl}_{3}, \delta\right): 141.5(\times 2), 129.3(\times 2), 128.4(\times 4), 126.0,124.9,123.4,109.6,108.9,108.7$, 99.4, 88.7, 36.5, 32.8, 31.0, 22.1, 16.1, 14.0, 13.7. MS m/ $z$ (relative intensity): 330 ( $\left.\mathrm{M}^{+}, 23\right)$, 239 (100), 209 (21), 91 (11). HRMS (EI) calcd for $\mathrm{C}_{23} \mathrm{H}_{26} \mathrm{~N}_{2}\left(\mathrm{M}^{+}\right) 330.2096$, found 330.2080 .

\section{Representative Procedure for Exhaustive Reduction of Bis-pyrrolopyrimidines (12a, T6)}

$\mathrm{PtO}_{2}(23 \mathrm{mg})$ and $\mathrm{HBr}\left(235 \mu \mathrm{L}, 2.1 \mathrm{mmol}, 48 \%\right.$ in $\left.\mathrm{H}_{2} \mathrm{O}\right)$ were added to the solution of $\mathbf{4 d}$ $(230 \mathrm{mg}, 1.02 \mathrm{mmol})$ in $\mathrm{MeOH}(10 \mathrm{~mL})$. The mixture was stirred under hydrogen pressure (50 psi) for $40 \mathrm{~h}$. After this period, the mixture was filtered through Celite and concentrated under reduced pressure to give the amidinium salt 11a.

Without further purification, crude 11a was dissolved in anhydrous THF $(10 \mathrm{~mL})$. Molecular sieves $(4 \AA)(300 \mathrm{mg})$ were added to the mixture, stirred for $30 \mathrm{~min}$ and cooled to $0{ }^{\circ} \mathrm{C}$. $\mathrm{LiAlH}_{4}\left(5.0 \mathrm{~mL}, 1.0 \mathrm{M}\right.$ in THF) was added to the mixture dropwise at $0{ }^{\circ} \mathrm{C}$. The mixture was warmed to room temperature and stirred for $2 \mathrm{~h}$. After this period, saturated aqueous $\mathrm{Na}_{2} \mathrm{SO}_{4}$ was added to the mixture dropwise, and the white precipitate was filtered off through Celite. The filtrate was concentrated under reduce pressure and purified by silica 
gel chromatography with $5 \% \mathrm{EtOH} / \mathrm{CH}_{2} \mathrm{Cl}_{2}$ to give ( \pm )-tetraponerine $\mathrm{T} 6(\mathbf{1 2 a})$ as an oil (154 $\mathrm{mg}, 64 \%)$.

12a (T6). ${ }^{1} \mathrm{H}$ NMR $\left(500 \mathrm{MHz}, \mathrm{C}_{6} \mathrm{D}_{6}, \delta\right): 3.14(1 \mathrm{H}, \mathrm{m}), 3.02(1 \mathrm{H}, \mathrm{dt}, J=8.6,2.4 \mathrm{~Hz}), 2.98$ $(1 \mathrm{H}, \mathrm{t}, J=5.2 \mathrm{~Hz}), 2.52(1 \mathrm{H}, \mathrm{m}), 2.44(1 \mathrm{H}, \mathrm{m}), 2.05-1.98(2 \mathrm{H}, \mathrm{m}), 1.90-1.34(18 \mathrm{H}, \mathrm{m}), 0.99$ $(3 \mathrm{H}, \mathrm{t}, J=7.2 \mathrm{~Hz}) .{ }^{13} \mathrm{C}$ NMR $\left(125 \mathrm{MHz}, \mathrm{C}_{6} \mathrm{D}_{6}, \delta\right): 83.3,64.1,59.6,49.1,45.7,34.7,33.3$, 32.6, 30.6, 29.2, 25.9, 23.1, 21.3, 21.0, 14.4. MS $\mathrm{m} / z$ (relative intensity): $235\left(\mathrm{M}^{+}-1,100\right)$, 179 (87), 138 (81), 96 (81), 70 (52). HRMS (EI) calcd for $\mathrm{C}_{15} \mathrm{H}_{27} \mathrm{~N}_{2}\left(\mathrm{M}^{+}-1\right) 235.2174$, found 235.2163 .

12b (Crude). ${ }^{1} \mathrm{H}$ NMR $\left(500 \mathrm{MHz}, \mathrm{C}_{6} \mathrm{D}_{6}, \delta\right): 2.71(2 \mathrm{H}, \mathrm{m}), 2.44(1 \mathrm{H}, \mathrm{m}), 2.36-2.32(1 \mathrm{H}$, m), $2.0(1 \mathrm{H}, \mathrm{m}), 1.90-1.65(9 \mathrm{H}, \mathrm{m}), 1.54-1.25(17 \mathrm{H}, \mathrm{m}), 1.03-0.89(9 \mathrm{H}, \mathrm{m}) .{ }^{13} \mathrm{C}$ NMR $(125$ $\left.\mathrm{MHz}, \mathrm{C}_{6} \mathrm{D}_{6}, \delta\right): 87.7,66.7,62.5,61.2,58.6,42.2,39.3,36.7,35.5,32.5,30.3,30.2,29.8$, 29.5, 28.7, 25.7, 22.9, 19.6, 14.5, 14.4, 14.1. MS $\mathrm{m} / z$ (relative intensity): $319\left(\mathrm{M}^{+}-1,11\right)$, 263 (16), 152 (100), 82 (32). HRMS (EI) calcd for $\mathrm{C}_{21} \mathrm{H}_{39} \mathrm{~N}_{2}\left(\mathrm{M}^{+}-1\right) 319.3113$, found 319.3089 .

12c. ${ }^{1} \mathrm{H}$ NMR (500 MHz, $\left.\mathrm{C}_{6} \mathrm{D}_{6}, \delta\right): 2.63(1 \mathrm{H}, \mathrm{dd}, J=10.0,3.9 \mathrm{~Hz}), 2.58-2.54(1 \mathrm{H}, \mathrm{m}), 2.51$ $(1 \mathrm{H}, \mathrm{dq}, J=6.8,2.6 \mathrm{~Hz}), 2.40-2.34(1 \mathrm{H}, \mathrm{m}), 2.21(1 \mathrm{H}, \mathrm{ddd}, J=11.3,5.0,2.8 \mathrm{~Hz}), 1.83-$ $1.45(13 \mathrm{H}, \mathrm{m}), 1.19(3 \mathrm{H}, \mathrm{d}, J=4.2 \mathrm{~Hz}), 1.17(3 \mathrm{H}, \mathrm{d}, J=4.3), 0.98(3 \mathrm{H}, \mathrm{t}, J=7.5 \mathrm{~Hz}), 0.96$ $(3 \mathrm{H}, \mathrm{t}, J=7.5 \mathrm{~Hz}) .{ }^{13} \mathrm{C}$ NMR $\left(125 \mathrm{MHz}, \mathrm{C}_{6} \mathrm{D}_{6}, \delta\right): 89.0,71.3,62.6,62.0,59.9,38.1,32.1$, 30.6, 29.8, 29.1, 28.1, 26.3, 19.8, 10.2, 10.0, 8.0. MS m/ $Z$ (relative intensity): 249 ( $\mathrm{M}^{+}-1$, 36), 125 (20), 96 (100), 82 (24). HRMS (EI) calcd for $\mathrm{C}_{16} \mathrm{H}_{29} \mathrm{~N}_{2}\left(\mathrm{M}^{+}-1\right) 249.2331$, found 249.2327 .

5. ${ }^{1} \mathrm{H}$ NMR (500 MHz, $\left.\mathrm{CDCl}_{3}, \delta\right): 6.93(1 \mathrm{H}, \mathrm{s}), 6.56(1 \mathrm{H}, \mathrm{d}, J=3.8 \mathrm{~Hz}), 6.27(1 \mathrm{H}, \mathrm{d}, J=3.8$ $\mathrm{Hz}), 3.31(2 \mathrm{H}, \mathrm{t}, J=7.6 \mathrm{~Hz}), 2.58(2 \mathrm{H}, \mathrm{t}, J=7.6 \mathrm{~Hz}), 2.52(2 \mathrm{H}, \mathrm{t}, J=7.3 \mathrm{~Hz}), 1.76(2 \mathrm{H}$, sext, $J=7.6 \mathrm{~Hz}), 1.68-1.66(4 \mathrm{H}, \mathrm{m}), 1.49(2 \mathrm{H}, \mathrm{sext}, J=7.6 \mathrm{~Hz}), 1.34-1.32(4 \mathrm{H}, \mathrm{m}), 1.00$ $(3 \mathrm{H}, \mathrm{t}, J=7.3 \mathrm{~Hz}), 0.95(3 \mathrm{H}, \mathrm{t}, J=7.4 \mathrm{~Hz}), 0.88(3 \mathrm{H}, \mathrm{t}, J=7.0 \mathrm{~Hz}) .{ }^{13} \mathrm{C}$ NMR $(125 \mathrm{MHz}$, $\mathrm{CDCl}_{3}$, o): 142.9, 134.0, 131.1, 126.3, 115.5, 110.1, 97.7, 95.3, 76.6, 36.5, 31.5, 30.5, 29.7, 28.9, 23.4, 22.6, 22.3, 19.6, 13.9, 13.6. MS m/z (relative intensity): $310\left(\mathrm{M}^{+}, 33\right), 281$ (100), 254 (10), $182(19)$.

9. ${ }^{1} \mathrm{H}$ NMR (400 MHz, $\left.\mathrm{CDCl}_{3}, \delta\right): 7.06(1 \mathrm{H}, \mathrm{s}), 2.67(2 \mathrm{H}, \mathrm{t}, J=9.6 \mathrm{~Hz}), 2.43(2 \mathrm{H}, \mathrm{t}, J=8.8$ $\mathrm{Hz}), 1.68(2 \mathrm{H}$, quint, $J=9.4 \mathrm{~Hz}), 2.59(2 \mathrm{H}$, quint, $J=9.0 \mathrm{~Hz}), 1.43(2 \mathrm{H}$, sext, $J=9.6 \mathrm{~Hz})$, $1.33-1.28(4 \mathrm{H}, \mathrm{m}), 0.90(3 \mathrm{H}, \mathrm{t}, J=9.2 \mathrm{~Hz}), 0.86(3 \mathrm{H}, \mathrm{t}, J=8.5 \mathrm{~Hz}) .{ }^{13} \mathrm{C} \mathrm{NMR}(100 \mathrm{MHz}$, $\mathrm{CDCl}_{3}$, $\delta$ ): $174.5,160.9,153.2,120.5,98.1,78.2,37.5,31.4,29.9,28.4,22.4,22.0,19.1$, 13.9, 13.5. MS m/ $z$ (relative intensity): $263\left(\mathrm{M}^{+}-1,1\right), 235$ (11), 221 (23), 208 (100).

10. ${ }^{1} \mathrm{H}$ NMR $\left(500 \mathrm{MHz}, \mathrm{CDCl}_{3}, \delta\right): 6.90(1 \mathrm{H}, \mathrm{s}), 6.58(1 \mathrm{H}, \mathrm{d}, J=3.8 \mathrm{~Hz}), 6.31(1 \mathrm{H}, \mathrm{d}, J=$ $3.8 \mathrm{~Hz}), 3.22(2 \mathrm{H}, \mathrm{t}, J=7.6 \mathrm{~Hz}), 2.56(2 \mathrm{H}, \mathrm{t}, J=7.5 \mathrm{~Hz}), 1.76(2 \mathrm{H}, \mathrm{sext}, J=7.5 \mathrm{~Hz}), 1.70$ $1.64(2 \mathrm{H}, \mathrm{m}), 1.37-1.30(4 \mathrm{H}, \mathrm{m}), 1.01(3 \mathrm{H}, \mathrm{t}, J=7.3 \mathrm{~Hz}), 0.90(3 \mathrm{H}, \mathrm{t}, J=7.0 \mathrm{~Hz}) .{ }^{13} \mathrm{C}$ NMR (125 MHz, $\mathrm{CDCl}_{3}$, o): 143.3, 136.4, 135.8, 127.4, 117.1, 109.7, 99.5, 36.4, 32.1, 31.8, 29.0, 24.4, 23.0, 14.5, 14.2. MS m/ $z$ (relative intensity): $264\left(\mathrm{M}^{+}, 14\right), 235$ (100), 178 (14).

\section{Supplementary Material}

Refer to Web version on PubMed Central for supplementary material.

\section{Acknowledgments}

We gratefully acknowledge the financial support of the National Institutes of Health (GM-64444). 


\section{References}

1. (a) Merlin P, Braekman JC, Daloze D, Pasteels JM. J Chem Ecol. 1988; 14:517.(b) Braekman JC, Daloze D, Pasteels JM, Vanhecke P, Declercq JP, Sinnwell V, Franke WZ. Naturforsch, C: Biosci. 1987; 42c:627.

2. (a) Stragies R, Blechert SJ. Am Chem Soc. 2000; 122:9584.(b) Plehiers M, Heilporn S, Ekelmans D, Leclercq S, Sangermano M, Braekman JC, Daloze D. Can J Chem. 2000; 78:1030.(c) Yue C, Gauthier I, Royer J, Husson HP. J Org Chem. 1996; 61:4949.(d) Devijver C, Macours P, Braekman JC, Daloze D, Pasteels JM. Tetrahedron. 1995; 51:10913.

3. Kim JT, Gevorgyan V. Org Lett. 2002; 4:4697. [PubMed: 12489964]

4. Kel'in AV, Sromek AW, Gevorgyan V. J Am Chem Soc. 2001; 123:2074. [PubMed: 11456838]

5. (a) Sonogashira K, Tohda Y, Hagihara N. Tetrahedron Lett. 1975:4467.(b) Sonogashira, K. Comprehensive Organic Synthesis. Trost, BM.; Fleming, I., editors. Vol. 3. Pergamon Press; New York: 1991. p. 521

6. For the syntheses of dibromopyrimidines, see Experimental Section. Janin YL, Roulland E, Beurdeley-Thomas A, Decaudin D, Monneret C, Poupon MF. J Chem Soc Perkin Trans 1. 2002:529.

7. For selective mono cross-coupling reactions of pyrimidine dihalides, see: Tullis JS, VanRens JC, Natchus MG, Clark MP, De B, Hsieh LC, Janusz MJ. Bioorg Med Chem Lett. 2003; 13:1665. [PubMed: 12729637] Simkovsky NM, Ermann M, Roberts SM, Parry DM, Baxter AD. J Chem Soc, Perkin Trans 1. 2002:1847.Gong Y, Pauls HW. Synlett. 2000:829.Mangalagiu I, Benneche T, Undheim K. Acta Chem Scand. 1996; 50:914.

8. For lower thermal stability of the terminal allenic intermediates, see ref 1 and: Kel'in AV, Gevorgyan V. J Org Chem. 2002; 67:95. [PubMed: 11777444]

9. For hydrogenation of pyrimidines, see: Brown DJ. The Pyrimidines. The Chemistry of Heterocyclic Compounds. John Wiley \& SonsNew York1994; 52:790-793.Brown DJ. The Pyrimidines Supplement I. The Chemistry of Heterocyclic Compounds. John Wiley \& SonsNew York1970:337341.

10. ${ }^{13} \mathrm{C}$ NMR analysis of the crude material revealed that $11 \mathrm{~d}$ was formed as a single diastereomer. The relative configuration of its stereogenic centers was assigned by NOE experiment after the subsequent reduction step.

11. For stereoelectronic control in addition of nucleophiles to an amidinium ion, see: Perrin CL, Young DB. J Am Chem Soc. 2001; 123:4451. [PubMed: 11457230] Fülöp F, Simon K, Tóth G, Hermecz I, Mészáros Z, Bernáath G. J Chem Soc, Perkin Trans 1. 1982; 12:2801.Kirby AJ. Stereoelectronic effects. Oxford Chemistry Primer. 36Oxford University PressOxford1996; :54-55.Barluenga J, Tomás M, Kouznetsov V, Rubio E. J Org Chem. 1994; 59:3699.

12. (a) Yanai M, Naito TJ. Pharm Soc Jpn. 1941; 61:99.(b) Huckin SN, Weiler L. J Am Chem Soc. 1974; 96:1082.(c) Botta M, Cavalieri M, Ceci D, Angelis FD, Finizia G, Nicoletti R. Tetrahedron. 1984; 40:3313.(d) Danel K, Larsen E, Pedersen EB. Synthesis. 1995:934. 
favored face of hydride attack

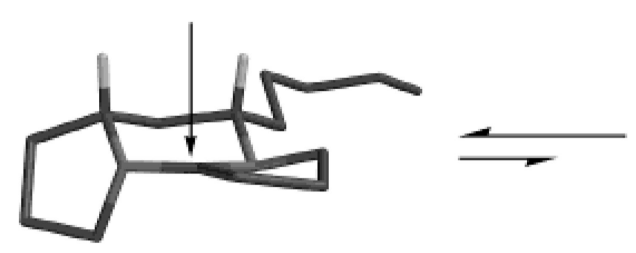

11a-eq

(22.4 Kcal/mol) favored face of hydride attack

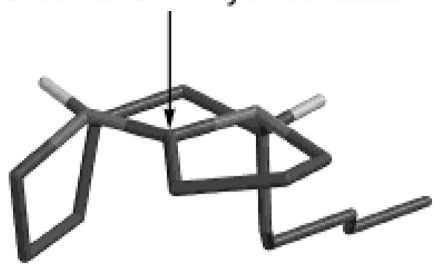

11a-ax

$(24.8 \mathrm{Kcal} / \mathrm{mol})$

Figure 1.

Two major conformations of amidinium ion 11a. 

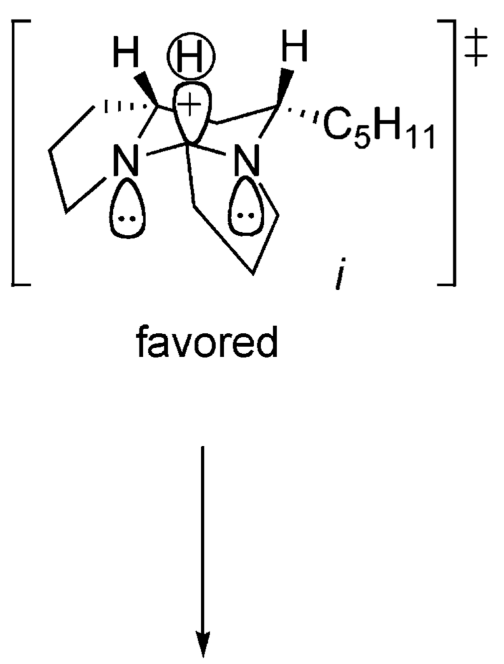

$( \pm)-\mathrm{T} 6$

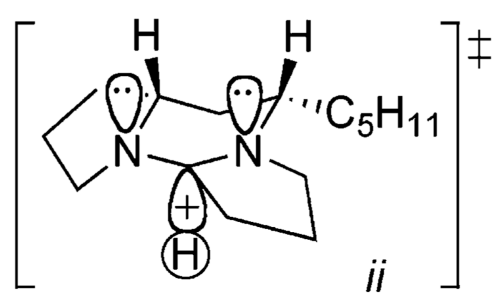

disfavored

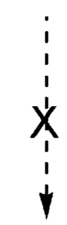

epi ( \pm$)-T 6$

\section{not observed}

Figure 2.

Proposed transition states for the nucleophilic attack of hydride at amidinium ion 11a. 


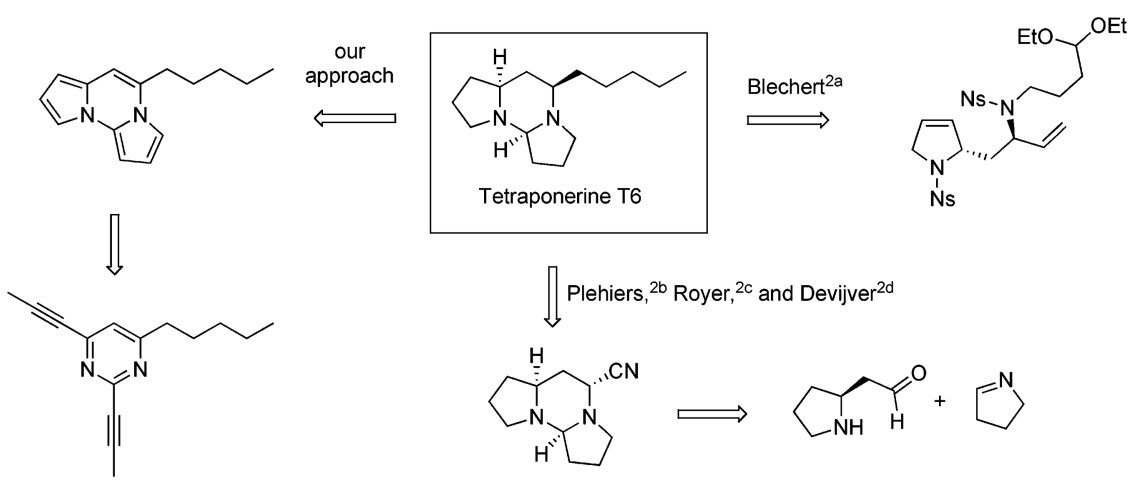

Scheme 1. Approaches toward Tetraponerine T6 


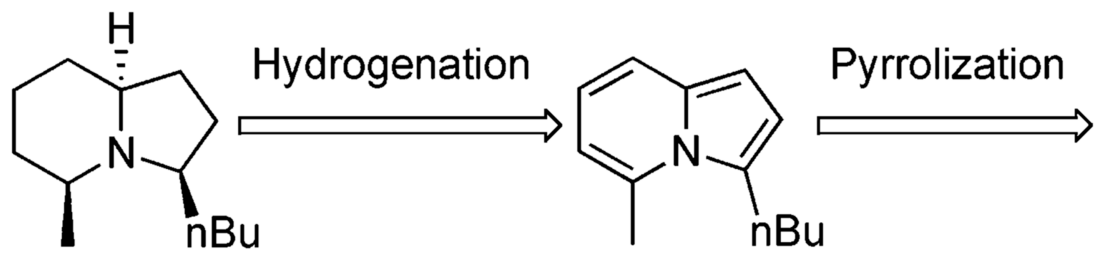

(士)-Monomorine

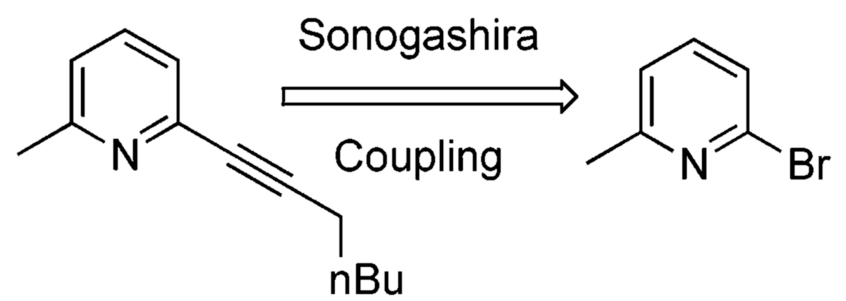

Scheme 2. Short Synthesis of ( \pm )-Monomorine 


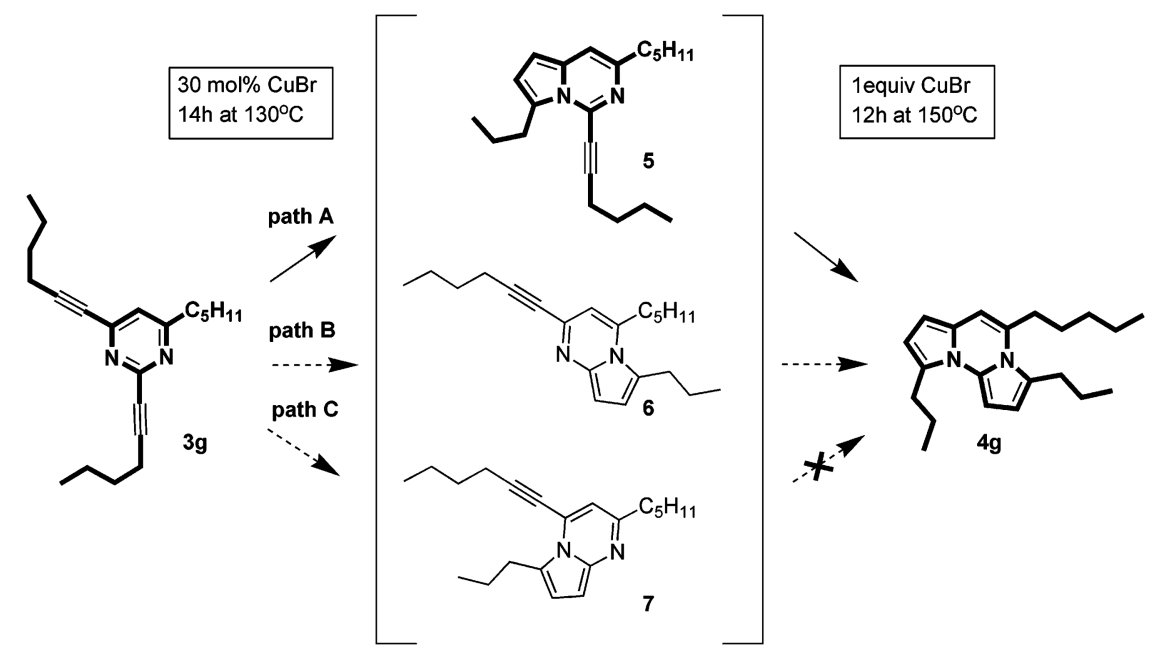

Scheme 3. Possible Pathways toward Bis-pyrrolopyrimidine 4g 

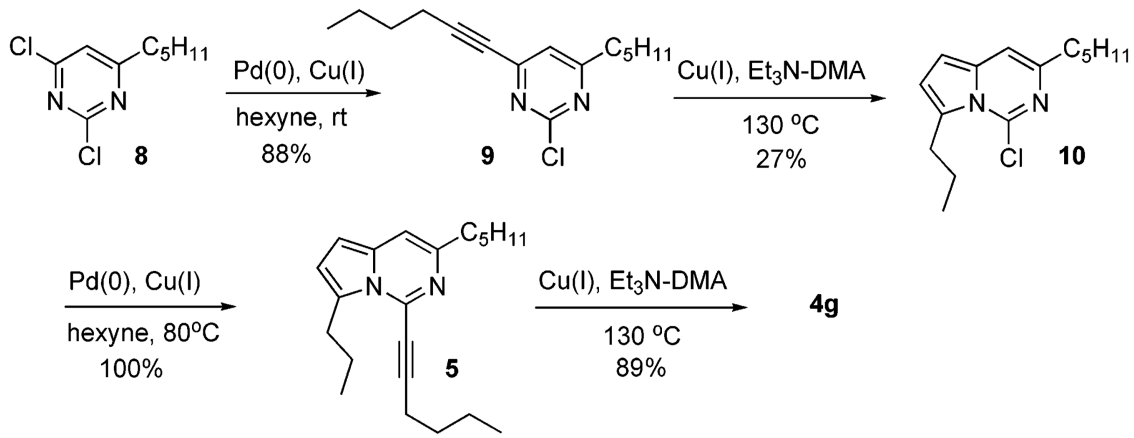

Scheme 4. Synthesis of Mono-pyrrolopyrimidine Intermediate 5 


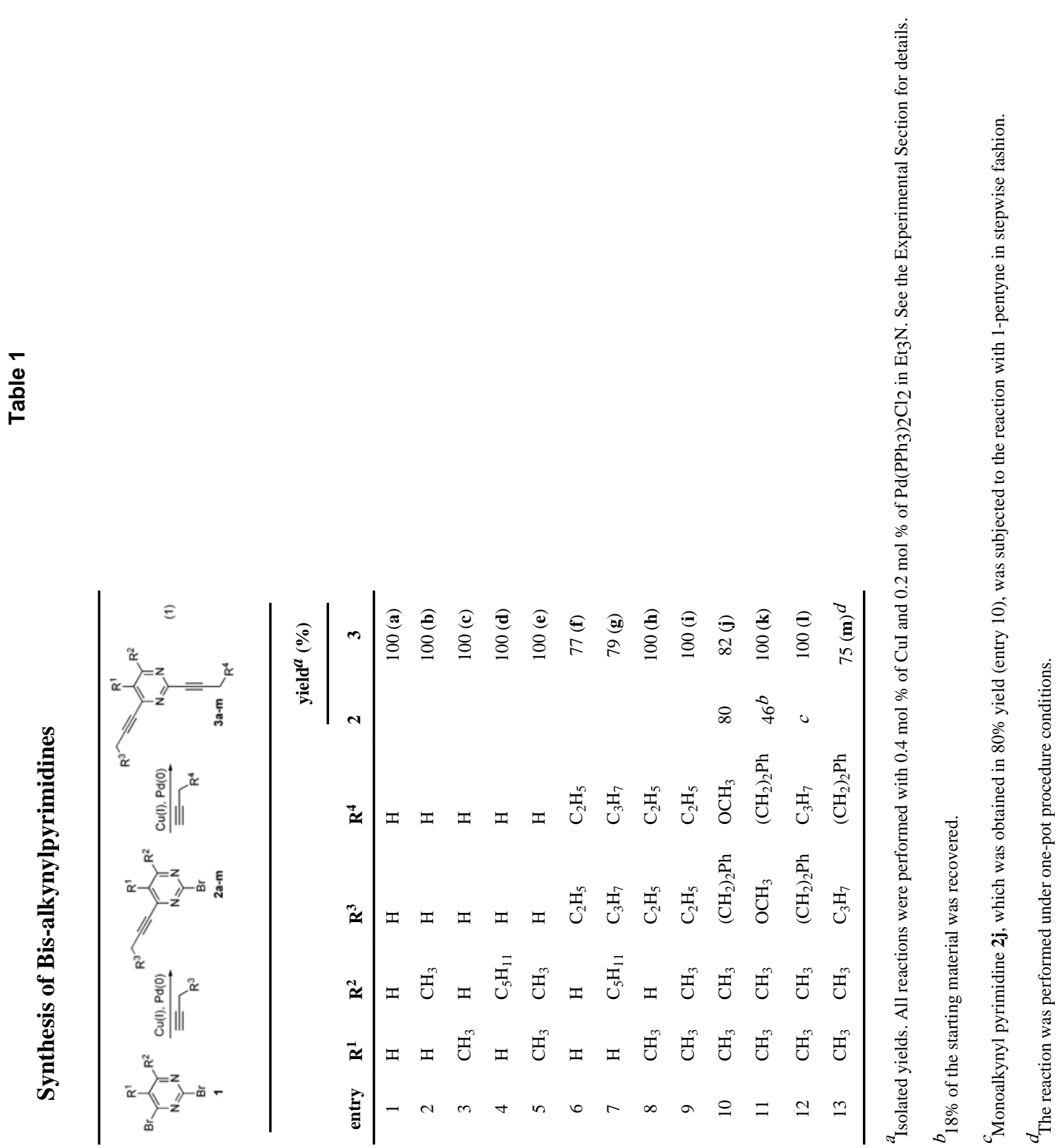


